\title{
Guidance for the implementation of a safety checklist for gastrointestinal endoscopic procedures: European Society of Gastrointestinal Endoscopy (ESGE) and European Society of Gastroenterology and Endoscopy Nurses and Associates (ESGENA) Position Statement
}

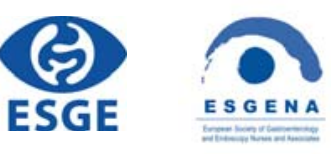

Authors

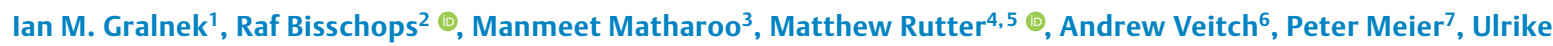
Beilenhoff $^{8}$, Cesare Hassan ${ }^{9,10}$, Mario Dinis-Ribeiro ${ }^{11}$, Helmut Messmann ${ }^{12}$

Institutions

1 Ellen and Pinchas Mamber Institute of Gastroenterology and Hepatology, Emek Medical Center, Afula, and Rappaport Faculty of Medicine Technion Israel Institute of Technology, Haifa, Israel

2 Department of Gastroenterology and Hepatology, Catholic University of Leuven (KUL), TARGID, University Hospitals Leuven, Leuven, Belgium

3 Wolfson Unit for Endoscopy, St. Mark's Hospital, London, UK

4 North Tees and Hartlepool NHS Foundation Trust, Stockton-on-Tees, UK

5 Population Health Sciences Institute, Newcastle University, Newcastle, UK

6 Department of Gastroenterology, Royal Wolverhampton NHS Trust, Wolverhampton, United Kingdom

7 Med. Klinik II, Diakovere Henriettenstift, Klinik für Enterologie, Hannover, Germany

8 Ulm, Germany

9 Department of Biomedical Sciences, Humanitas University, Pieve Emanuele, Milan, Italy

10 Endoscopy Unit, IRCCS Humanitas Research Hospital, Milan, Italy
11 Porto Comprehensive Cancer Center (Porto.CCC) \& RISE@CI-IPOP (Health Research Network), Porto, Portugal

12 III. Medizinischen Klinik, Klinikum Augsburg, Augsburg, Germany

published online 14.12 .2021

Bibliography

Endoscopy 2022; 54: 206-210

DOI 10.1055/a-1695-3244

ISSN 0013-726X

(C) 2021. European Society of Gastrointestinal Endoscopy

All rights reserved.

This article is published by Thieme.

Georg Thieme Verlag KG, Rüdigerstraße 14,

70469 Stuttgart, Germany

Corresponding author

Helmut Messman, MD, III. Medizinischen Klinik, Klinikum

Augsburg, Stenglinstrasse 2, 86156 Augsburg, Germany

Fax: +49-821-4003331

helmut.messmann@klinikum-augsburg.de

\section{Introduction}

Continuous quality improvement and patient safety in gastrointestinal (GI) endoscopy are overarching priorities of the European Society of Gastrointestinal Endoscopy (ESGE) and the European Society of Gastroenterology and Endoscopy Nurses and Associates (ESGENA). Consistently with these societal priorities, ESGE has developed and disseminated quality indicators to improve both the efficacy and safety of GI endoscopy procedures $[1,2]$. Moreover, the patients we serve expect us to pro- vide a safe, rigorous, and standardized approach to the performance of both diagnostic and therapeutic GI endoscopy procedures.

Since Haynes et al. published their landmark study in 2009 [3], reporting that a surgery safety checklist (the World Health Organization [WHO] Surgical Safety Checklist) significantly reduced postoperative surgical mortality and inpatient adverse events, there has been a growing body of evidence demonstrating the importance of surgical safety checklists in the operating 
theatre $[4,5]$. Studies have shown that safety checklists in the operating theatre enhance a team approach, flatten hierarchies, improve team communication and nontechnical skills, and contribute to the safe delivery of patient care [4,5]. Taken altogether, this has led to the uptake and routine use of surgery safety checklists in operating theatres around the world.

ESGE and ESGENA believe that these same principles apply to the field of $\mathrm{Gl}$ endoscopy. This is particularly relevant given the burgeoning volume of endoscopic procedures that are increasingly interventional and technically advanced, coupled with an aging patient population with more comorbidities. Considering this evolution in endoscopic practice, patient safety must not be compromised and measures to maintain and improve safety in $\mathrm{Gl}$ endoscopy should continually be sought [6]. Given the above, the introduction and use of Gl endoscopy safety checklists has gained traction in recent years [6-10].

Despite the recognized importance of Gl endoscopy safety checklists, there are limited data on their implementation or actual use in practice in GI endoscopy units around the world. Moreover, there are still no high-level data showing that endoscopy safety checklists improve patient safety in the Gl endoscopy unit, including rates of mortality, adverse events, or endoscopy completion. This evidence base is difficult to obtain given the relative rarity of severe adverse events in $\mathrm{Gl}$ endoscopy. In a recent systematic review and narrative synthesis on checklist feasibility and impact on GI endoscopy, Bitar and colleagues reported that endoscopy team communication and teamwork significantly improved with the implementation of a $\mathrm{Gl}$ endoscopy safety checklist [10]. Although most published studies evaluating safety checklists in the GI endoscopy setting have not reported on associations between checklist implementation and clinical outcomes, it may be extrapolated that with improved team communication, medical errors may be reduced and adverse events thereby prevented $[10,11]$.

\section{Methods}

To identify published biomedical literature on this topic, a Pubmed/MEDLINE search was performed using "surgery," "endoscopy," "gastrointestinal endoscopy," "digestive system endoscopy," "gastrointestinal endoscopic examination," "safety," and "checklist" as MeSH terms.

\section{What is a Gl endoscopy safety checklist?}

Analogously to surgical safety checklists, GI endoscopy safety checklists comprise three distinct, yet equally important phases: "Sign in," "Time out," and "Sign out" $[3,10]$.

1. The "Sign in" phase occurs once the patient enters the endoscopy room. This phase includes:

a) Introduction to the patient of the endoscopy team (including names and roles) and, where relevant, including the anesthesiologist

b) Verification of patient identity

c) Completion and verification of the appropriate informed consent form(s) d) Documentation of the patient's American Society of Anesthesiologists (ASA) score. GI endoscopy units that use moderate sedation/general anesthesia may also document the patient's Mallampati score [12]

e) A review of pertinent medical/surgical comorbidities (including cardiopulmonary risks, presence of cardiac pacemakers/defibrillators, and/or other implantable medical devices),

f) A review of medication use (including anticoagulant/antiplatelet medications)

g) A review of known drug allergies, including any difficulties with previous sedation/anesthesia

h) A review of dental status (e. g., loose teeth, dentures, bridge)

i) A review of known communicable patient infections (e. g., hepatitis B virus [HBV], hepatitis C virus [HCV], human immunodeficiency virus [HIV], tuberculosis [TB], COVID, etc)

j) Confirmation of the pre-endoscopy preparation (e.g. nil per os and/or correct bowel preparation)

2. The "Time out" phase occurs immediately prior to the induction of moderate sedation/general anesthesia or insertion of the endoscope in nonsedated procedures. This phase includes:

a) Confirmation of the planned Gl endoscopic procedure(s) (including indications, aims, and potential limitations)

b) Confirmation that all required/appropriate endoscope(s), endoscopic accessories, and ancillary equipment are available and functioning correctly

c) Confirmation of functioning intravenous access, where indicated

d) Confirmation that the appropriate patient monitoring equipment is prepared and functioning correctly (including pulse oximetry, blood pressure, and cardiac monitor)

e) Confirmation that information on relevant comorbidities and on limitations concerning patient sedation and airway management has been shared amongst the endoscopy team

f) Confirmation that antibiotic prophylaxis and/or preendoscopic retrograde cholangiopancreatography (ERCP) nonsteroidal anti-inflammatory drug (NSAID) has been given if clinically indicated

3. The "Sign out" phase occurs after the completion of the endoscopic procedure(s), but prior to the patient's exiting the endoscopy room. This phase includes:

a) Confirmation that all histological samples taken during endoscopy are present, correctly labelled, documented, and cross-checked by both the endoscopist and the assisting nurse

b) Confirmation that the endoscopy report is accurate, including post-procedure patient instructions and any follow-up procedures that may be indicated. 


\section{ESGE-ESGENA GI endoscopy safety checklist*}

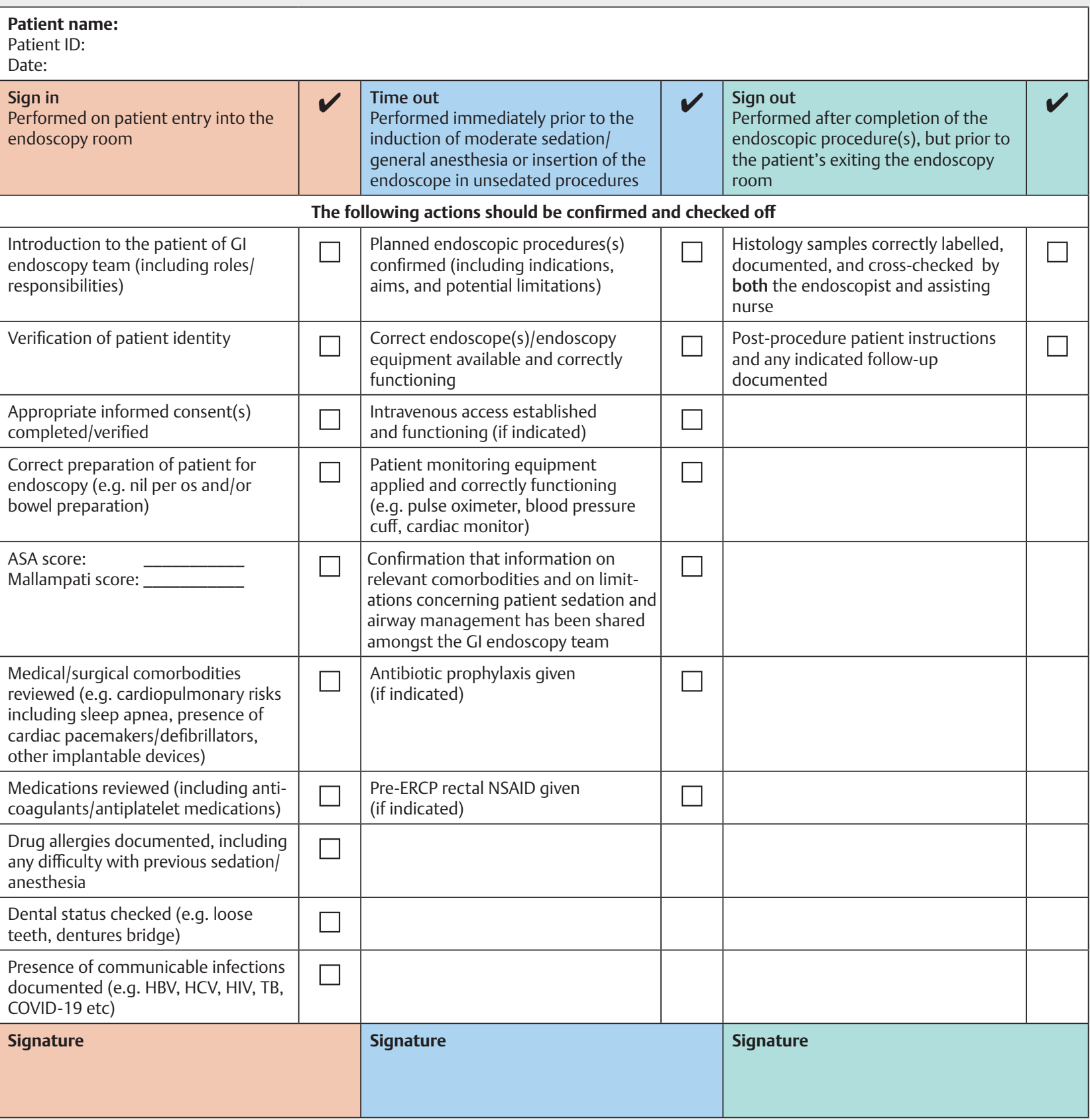

ASA, American Society of Anesthesiologists; ERCP, endoscopic retrograde cholangiopancreatography; ESGE, European Society of Gastrointestinal Endoscopy; ESGENA, European Society of Gastroenterology and Endoscopy Nurses and Associates; Gl, gastrointestinal; HBV, hepatitis B virus; HCV, hepatitis C virus; HIV, human immunodeficiency virus; NSAID, nonsterodial anti-inflammatory drug; TB, tuberculosis

*This checklist is not intended to be comprehensive. Modifications to fit local $\mathrm{Gl}$ endoscopy practice are allowed.

- Fig. 1 European Society of Gastrointestinal Endoscopy and European Society of Gastroenterology and Endoscopy Nurses and Associates (ESGE-ESGENA) Gastrointestinal Endoscopy Safety Checklist. This checklist is not intended to be comprehensive and can be adapted to local gastrointestinal endoscopy practice. 


\section{Safety checklist implementation}

With respect to the practical implementation of the $\mathrm{Gl}$ endoscopy safety checklist, ESGE and ESGENA recommend that a single individual from the endoscopy team takes the lead [6]. This same individual should continue to have the lead throughout the day's endoscopy procedure list, and can be the endoscopist or the endoscopy nurse. However, as with all aspects of $\mathrm{Gl}$ endoscopy, from patient consent to technical outcome, the ultimate responsibility resides with the lead endoscopist. Irrespective of who leads the checklist process, it is important that the entire endoscopy team is actively engaged in and contributing to all three phases of the GI endoscopy safety checklist. For example, during the "Time out" phase, no competing activities should be undertaken in the endoscopy room and distractions minimized (e.g., by locking the door to prevent interruptions and/or queries about other patients).

As the field of Gl endoscopy rapidly becomes more akin to "endoscopic surgery," ESGE and ESGENA strongly recommend the implementation of safety checklists as part of the standard practice for all Gl endoscopy procedures. This will require adapting and adopting successful checklist strategies from the surgical operating theatre but making them specific and relevant to $\mathrm{Gl}$ endoscopic practice. We here provide a generic $\mathrm{Gl}$ endoscopy safety checklist for use ( $\triangleright$ Fig.1). This safety checklist can be adapted as dictated by local needs and requirements and translated into local languages. Once a generic $\mathrm{Gl}$ endoscopy checklist has been successfully implemented, endoscopy units may wish to further devise procedure-specific checklists (e.g., for ERCP, endoscopic ultrasound [EUS], percutaneous endoscopic gastrostomy [PEG]) to enhance safety practices.

To be successful, checklist implementation requires a considered approach with education and engagement from key stakeholders. It should be noted that paper-based checklists may be initially easier to implement, but their data are more difficult to evaluate and report. Electronic checklists may be included in the electronic $\mathrm{Gl}$ endoscopy reporting systems; these enable easier data evaluation for quality assurance and/or scientific purposes and provide more definitive medicolegal documentation.

\section{Overcoming potential barriers}

ESGE and ESGENA realize there may be barriers to the implementation and routine use of endoscopy safety checklists in GI endoscopy. These barriers may include a lack of patient safety culture, negative attitudes/resistance to change, and additions to the endoscopic procedure leading to inefficiencies, as well as a perceived loss of medical autonomy. These barriers were also faced by the surgical community and lessons can be learned for Gl endoscopy. Barriers to checklist adoption can be minimized by having a clear checklist training strategy for endoscopists, endoscopy nurses, and managerial teams, so there is a unified understanding of the benefits of checklists, not only for safety and quality but also for GI endoscopy unit efficiency. Mandating checklists without an implementation strategy that has been created in concert with the end-users may hinder this process.
Moreover, if Gl endoscopy safety checklists are not well designed with content validity, and therefore contain irrelevant items, they will be perceived as a waste of time and as a simple "tick-box" exercise.

These potential barriers will need to be overcome with hard work and demonstrated senior leadership by both the endoscopists and the nursing staff of the GI endoscopy unit. Thus, to facilitate the implementation of an endoscopy safety checklist, we recommend formal education of the entire GI endoscopy team on the importance of patient safety and endoscopy quality; the endoscopy checklist is one tool in the armamentarium for delivering this. Such practical measures of implementing safety checklists will feed into a culture of patient safety in the $\mathrm{Gl}$ endoscopy unit over time.

Recommended also are: endorsement from senior endoscopy unit leadership by championing the checklist; a training plan for the entire Gl endoscopy team; a target for the checklist adherence rate (e.g. $\geq 80 \%$ ); support for those who adopt and routinely use the checklist; debriefing for those who are noncompliant; continued reassessment and audit; and targeted feedback on checklist use $[6,10]$. It may be helpful to follow a PDAS (Plan, Do, Act, Study) cycle framework as recommended by Bitar et al. [10].

These facilitating activities will need to be emphasized and optimized if we, as $\mathrm{Gl}$ endoscopists and endoscopy nurses, are to be successful in advancing patient safety in the GI endoscopy unit and thereby improving the overall quality of patient care.

\section{Competing interests}

M. Dinis-Ribeiro has provided teaching consultancy to Boston Scientific (2019) and consultancy to Medtronic (2020); his department receives an educational grant from Olympus (ongoing) and a research grant from Fujifilm (ongoing); he receives a fee as a Co-Editor-inChief of Endoscopy. I.M. Gralnek has provided consultancy to Boston Scientific, Medtronic, Motus GI, Neurogastrx, Simbionix, and Vifor Pharma; he has received research funding from AMG Gastro, AstraZeneca, and CheckCap. C. Hassan has received research support (2020 to 2021) from Medtronic, Fujifilm, Alfasigma, and Norgine. H. Messman, in the past 3 years, has received benefits from the following endoscopy companies: Apollo Endosurgery, Biogen, Boston Scientific, CDx Diagnostic, Cook Medical, CSL Behring, Dr. Falk Pharma, Endo Tools Therapeutics, Erbe, Fujifilm, Hitachi, Janssen-Cilag, Medwork, Norgine, Nutricia, Olympus, Ovesco Endoscopy, Servier Deutschland, and US Endoscopy; he has received grants from Amgen, Bayer, Dr. Falk Pharma, MSD, Novartis, Olympus and Roche; he has received honoraria from Covidien, Dr. Falk Pharma, and Olympus, and consultancy fees from Boston Scientific, CDx Diagnostics, Covidien, Erbe, Lumendi, Norgine, and Olympus; he has received research support from Olympus (ESD-Register) and Satisfai. U. Beilenhoff, R. Bisschops, M. Matharoo, P. Meier, M. Rutter, and A. Veitch have no competing interests to declare. 


\section{References}

[1] Valori R, Cortas G, de Lange T et al. Performance measures for endoscopy services: a European Society of Gastrointestinal Endoscopy (ESGE) Quality Improvement Initiative. Endoscopy 2018; 50: 119

[2] Bisschops R, Rutter MD, Areia M et al. Overcoming the barriers to dissemination and implementation of quality measures for gastrointestinal endoscopy: European Society of Gastrointestinal Endoscopy (ESGE) and United European Gastroenterology (UEG) Position Statement. Endoscopy 2021; 53: 196-202

[3] Haynes AB, Weiser TG, Berry WR et al. A surgical safety checklist to reduce morbidity and mortality in a global population. $N$ Engl J Med 2009; 360: 491-499

[4] Borchard A, Schwappach DL, Barbir A et al. A systematic review of the effectiveness, compliance, and critical factors for implementation of safety checklists in surgery. Ann Surg 2012; 256: 925-933

[5] Treadwell JR, Lucas S, Tsou AY. Surgical checklists: a systematic review of impacts and implementation. BMJ Qual Saf 2014; 23: 299-318
[6] Matharoo M, Thomas-Gibson S, Haycock A et al. Implementation of an endoscopy safety checklist. Frontline Gastroenterol 2013; 5: 260265

[7] Dubois H, Schmidt PT, Creutzfeldt J et al. Person-centered endoscopy safety checklist: development, implementation, and evaluation. World J Gastroenterol 2017; 23: 8605-8614

[8] Kherad O, Restellini S, Menard C et al. Implementation of a checklist before colonoscopy: a quality improvement initiative. Endoscopy 2017; 50: 203-210

[9] Raphael K, Cerrone S, Sceppa E et al. Improving patient safety in the endoscopy unit: utilization of remote video auditing to improve timeout compliance. Gastrointest Endosc 2019; 90: 424-29

[10] Bitar V, Martel M, Restellini S et al. Checklist feasibility and impact in gastrointestinal endoscopy: a systematic review and narrative synthesis. Endosc Int Open 2021; 09: E453-E460

[11] Cripsin A, Birkner B, Munte A et al. Process quality and incidence of acute complications in a series of more than 230,000 outpatient colonoscopies. Endoscopy 2009; 41: 1018-1025

[12] ASGE Standards of Practice Committee. Guidelines for sedation and anesthesia in Gl endoscopy. Gastrointest Endosc 2018; 87: 327-337 